\title{
Coulomb explosion of methyl iodide clusters using giga watt laser pulses in the visible region: Effect of wavelength, polarisation and doping
}

\author{
S DAS, P SHARMA and R K VATSA* \\ Chemistry Division, Bhabha Atomic Research Centre, Mumbai 400085 \\ e-mail: rkvatsa@barc.gov.in
}

MS received 19 February 2009; 3 June 2009; accepted 10 June 2009

\begin{abstract}
Nanosecond laser-induced Coulomb explosion studies have been carried out for methyl iodide clusters at 532 and $563 \mathrm{~nm}$ under similar laser intensity $\left(\sim 5 \times 10^{9} \mathrm{~W} / \mathrm{cm}^{2}\right)$ conditions. Multiply charged atomic ions of carbon and iodine having large kinetic energy $(\sim 100 \mathrm{~s}$ of eV) were observed in both the cases. Observation of higher charged states at $563 \mathrm{~nm}$ for Coulomb exploded atomic ions supports the preposition of enhanced inverse bremsstrahlung heating of the ionized cluster system at this wavelength. The angular distribution of the multiply charged atomic ions is found to be isotropic with respect to laser polarization direction at $532 \mathrm{~nm}$. When water doped methyl iodide clusters were irradiated at $563 \mathrm{~nm}$, highly charged atomic ions of oxygen along with carbon and iodine were also observed. This result suggests that the mechanism leading to Coulomb explosion is a collective property of the cluster as a whole and individual molecular properties do not play significant role.
\end{abstract}

Keywords. Methyl iodide; clusters; Coulomb explosion; time-of-flight mass spectrometry; multiply charged ions; kinetic energy.

\section{Introduction}

Clusters represent a bridge between a molecular level and a bulk description of matter. ${ }^{1}$ Because of their high local electron density, they interact efficiently with laser radiation and serve as an attractive targets for studying several nonlinear phenomena including Coulomb explosion. ${ }^{2}$ Coulomb explosion of atomic and molecular clusters has been reported using laser pulses over a wide range of electromagnetic spectrum ranging from IR to X-ray region employing intensity in the range of $10^{14}-10^{20} \mathrm{~W} / \mathrm{cm}^{2} .^{3-7}$ These studies are motivated by the desire to develop table top accelerators, generate higher order harmonics, energetic electrons, multiply charged ions and even neutrons. ${ }^{8-11}$ Recently, Coulomb explosion has been reported using nanosecond laser pulses of intensity $\sim 10^{9} \mathrm{~W} / \mathrm{cm}^{2}$ in methyl iodide, carbon disulfide, acetone and several other molecular clusters leading to formation of multiply charged atomic ions (up to $\mathrm{C}^{4+}, \mathrm{I}^{5+}, \mathrm{S}^{6+}$ and $\mathrm{O}^{4+}$ ) with large kinetic energy. ${ }^{12-15}$ Generation of these multiply charged atomic ions at such low laser intensity is rather un-

\footnotetext{
*For correspondence
}

usual. Though the high intensity Coulomb explosion is reasonably well understood, the understanding of giga watt induced Coulomb explosion at present is very meagre. It is not clear as to how such low fields can give rise to formation of ions with high ionization potentials and large kinetic energies. There must be a highly efficient energy absorption mechanism which couples the optical field and the cluster. Clearly, more experimental and theoretical studies are needed to clarify several aspects of laser-matter interaction at these intensity levels.

In our previous studies, significance of intermediate excited electronic state and cluster size in initiating Coulomb explosion process in clusters was emphasized. ${ }^{12,14,15}$ Recently, a three-stage model comprising of 'multiphoton ionization ignited-inverse bremsstrahlung heating-electron impact ionization' has been proposed to explain different facets of Coulomb explosion phenomenon occurring on interaction of molecular clusters with nanosecond laser pulses with intensity $\sim 10^{9}-10^{11} \mathrm{~W} / \mathrm{cm}^{2} .{ }^{17,18}$ This model assumes that the nanosecond laser-induced Coulomb explosion of cluster is initiated by multiphoton ionisation (MPI) of the constituent molecules within the neutral clusters. Subsequently some 
of the ionized electrons, which still continue to form integral part of ionized cluster (i.e. caged electrons), extract energy from the laser field via inverse bremsstrahlung (IBS) process i.e. absorption of energy from the electromagnetic laser field on collision with neutral and ionic species within the cluster. Once these caged electrons gain enough energy to surpass the corresponding ionization potential of ion, multiple ionization process may start via stepwise electron impact ionization resulting in generation of multiply ionized species within the cluster. Finally, these multiply ionized clusters, which are in close proximity, undergo Coulomb explosion due to strong electrostatic repulsion resulting in generation of multiply charged atomic ions with large kinetic energies.

In order to throw more light and to test the preposition of laser-assisted electron heating of molecular cluster, in the present work, we have irradiated methyl iodide clusters at two different wavelengths (532 and $563 \mathrm{~nm}$ in the visible region) having similar laser intensity in the range of $\sim 10^{9} \mathrm{~W} / \mathrm{cm}^{2}$. These wavelengths were chosen since the photon energy of $532 \mathrm{~nm}$ is $6 \%$ higher than that of $563 \mathrm{~nm}$ photon. However, the ponderomotive energy (defined as $\left.U_{p}=\mathrm{e}^{2} I \lambda^{2} /\left(8 \pi^{2} \varepsilon_{0} m_{e} c^{3}\right)=9.33 \times 10^{-14} I \lambda^{2}\left(\mu \mathrm{m}^{2}\right),{ }^{4}\right)$ which determines the heating rate of electron via IBS, at these wavelengths is $12 \%$ more for $563 \mathrm{~nm}$ than $532 \mathrm{~nm}$ at an intensity of $5 \times 10^{9} \mathrm{~W} / \mathrm{cm}^{2}$. Thus, these two opposing factors apparently might have overall similar effects in interaction with the cluster at 532 and $563 \mathrm{~nm}$ wavelengths. However, if IBS is a major process for generation of highly charged ions, the cumulative effect of the rate of energy deposited within the clusters which is governed by the product of ponderomotive energy and the total effective electron-ion/neutral collision frequency in the time span of the laser pulse would be considerably different at these two wavelengths. Thus, although the number of photons required for ionization of molecules within the cluster would be same at both the wavelengths but the electron heating rate would be quite different. This should reflect either in the charged state of the atomic ions or in their kinetic energies as the wavelength changes from 532 to $563 \mathrm{~nm}$. In addition, two different sets of experiments were also carried out. In the first set, laser polarization with respect to time-of-flight (TOF) axis was changed to understand the polarization dependency of the Coulomb explosion process. In the second set, a molecule with higher ionization poten- tial $\left(\mathrm{H}_{2} \mathrm{O}, \quad \mathrm{IP}=12.622 \mathrm{eV}\right)$ was doped in methyl iodide cluster to check whether only $\left(\mathrm{CH}_{3} \mathrm{I}\right)_{n}$ undergo Coulomb Explosion or the dopant which is part of cluster also undergoes Coulomb explosion giving rise to multiply charged ions with large kinetic energy.

\section{Experimental}

Details of the experimental set-up have been described in our earlier publications ${ }^{12,14,15,19}$ and only a brief description is given here. Neutral methyl iodide clusters were generated via supersonic expansion of $\mathrm{CH}_{3} \mathrm{I}$ seeded in helium (1-5 atm) using a pulsed valve $(0.6 \mathrm{~mm}$ nozzle diameter and 300 microseconds pulse duration). The supersonic jet so produced was skimmed at a distance of $5 \mathrm{~cm}$ from the pulsed nozzle. Ionization and Coulomb explosion were carried out using second harmonic of a pulsed $\mathrm{Nd}$ : YAG nanosecond laser (Quantel, Model YG 980 E) as well as with the dye laser output at $563 \mathrm{~nm}$. The distance between skimmer and ionization region was $17 \mathrm{~cm}$. The ions so formed were accelerated and guided into a $100 \mathrm{~cm}$ field-free region using double focusing Wiley-McLaren assembly and detected using the Channel Electron Multiplier (CEM) detector. Typical voltages applied to repeller and extractor grids were $2650 \mathrm{~V}$ and $1000 \mathrm{~V}$, respectively. The ion signal from CEM was transferred to a digital oscilloscope for averaging and further processed on a computer. The mass resolution of the instrument is $\sim 300$. Ion signal was optimized by varying the delay between pulse-valve opening and laser firing so that the laser pulse interacts with the clustered portion of gas pulse. The polarization of $532 \mathrm{~nm}$ laser was rotated by placing a half wave plate $(\lambda / 2$ plate) in the path of laser beam for polarization dependent studies. The $0^{\circ}$ is defined as the direction of the laser polarization parallel to the TOF axis, with $90^{\circ}$ being perpendicular to the axis. Measurements were made from $0^{\circ}-360^{\circ}$, with a step size $45^{\circ}$ degrees.

For generation of $563 \mathrm{~nm}$, a Quantel dye laser [TDL 70] with Rhodamine 6G solution in ethanol was pumped by the second harmonic of the $\mathrm{Nd}$ : YAG laser. The pulse energy of the dye laser could be varied by changing the YAG laser energy. An iris was introduced just prior to entering in the ionisation chamber to make sure that central portion of the laser beam was used for these experiments. 


\section{Results}

Figures (1a) and (b) depict the time-of-flight mass spectra of methyl iodide clusters subjected to 532 and $563 \mathrm{~nm}$ laser pulses respectively. Figure la inset exhibits the larger cluster fragment ions observed in the mass spectra for Coulomb explosion studies at $532 \mathrm{~nm}$. Both the spectra were recorded under laser intensity of $\sim 5 \times 10^{9} \mathrm{~W} / \mathrm{cm}^{2}$. It is clear from these figures that methyl iodide clusters exhibit the phenomenon of Coulomb explosion at both these wavelengths resulting in generation of multiply charged atomic ions of iodine and carbon, along with other cluster fragment ions. However, it can be observed that for $532 \mathrm{~nm}$ (figure 1a) the highest charged state observed are limited up to $\mathrm{C}^{3+}$ and $\mathrm{I}^{3+}$, whereas in case of $563 \mathrm{~nm}$ the highest charged states observed span up to $\mathrm{C}^{4+}$ and $\mathrm{I}^{5+}$. This observation reveals that for a given intensity the phenomenon of Coulomb explosion has a higher propensity at $563 \mathrm{~nm}$ as compared to $532 \mathrm{~nm}$.

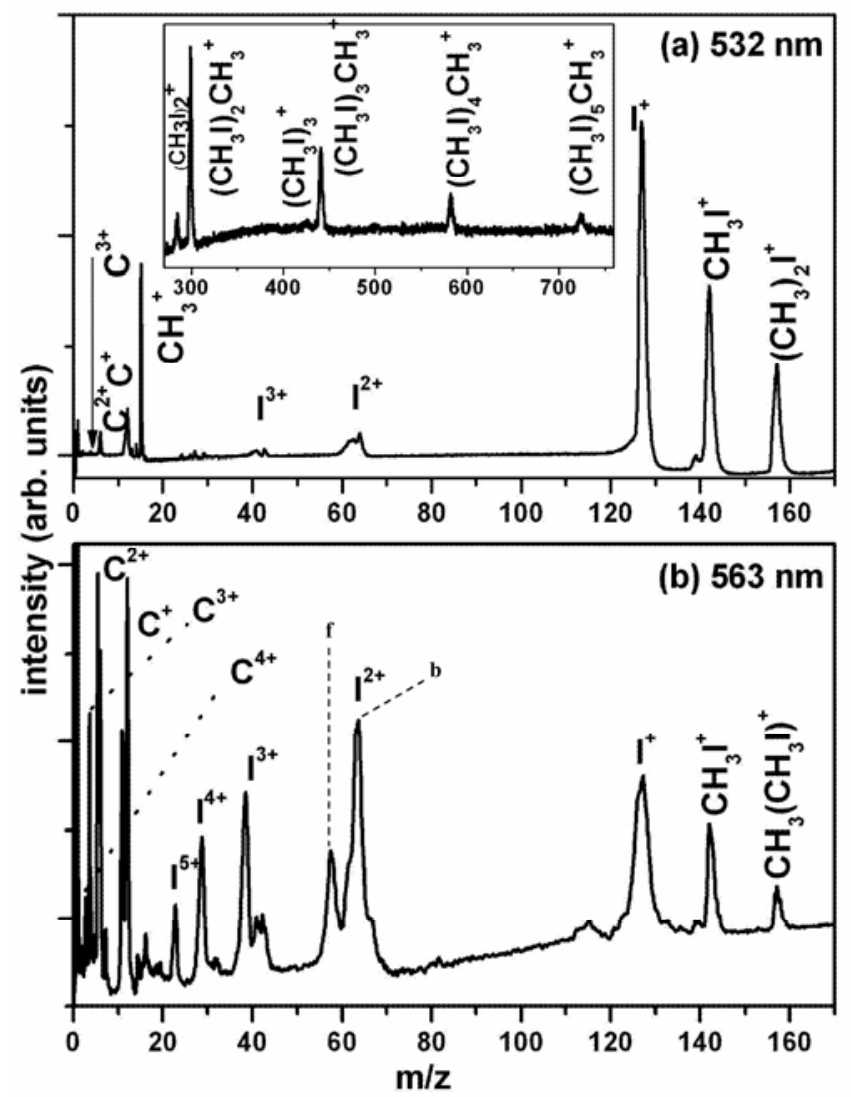

Figure 1. Time of flight mass spectra of methyl iodide clusters upon interaction with laser pulse of intensity $\sim 5 \times 10^{9} \mathrm{~W} / \mathrm{cm}^{2}$ at (a) $532 \mathrm{~nm}$ (f and b denote forward and backward ejected ions) and (b) $563 \mathrm{~nm}$. Inset shows higher cluster fragment ions detected at $532 \mathrm{~nm}$.
As can be seen, some of the ion signals show split peaks in figure 1 . The ions which are formed during the Coulomb explosion of methyl iodide clusters carry significant amount of kinetic energy and are liberated in all the directions. Those ions travelling along the time-of-flight axis towards the detector are unfocussed and arrive at the detector earlier. While the ions ejected in the opposite direction, i.e. away from the detector (towards the repeller plate of the ion source), are initially decelerated since these travel against the field, stopped and turned around in the TOF acceleration assembly and arrive at the detector at a later time resulting into the split of mass peak for a given ion.

The difference in arrival time of the forward and backward ions for a given mass can be used to calculate the kinetic energy of the ion using the following time-of-flight equation. ${ }^{12,14,15}$

$$
E_{\text {kin }}=9.65 \times 10^{-7} \Delta t^{2} n^{2} F^{2} /(8 m),
$$

where $\Delta t=$ time difference between the forward and backward mass peaks for a given ion (in nanosecond). $F=$ static electric field for ion extraction $\left.(\mathrm{V} \mathrm{cm})^{-1}\right), n=$ charge of the ion, $m=$ mass of the fragment (amu).

One must keep in mind that this measurement reflects the kinetic energy gained by the given charged ion due to Coulomb explosion only and the acceleration of the ions due to application of extraction field has no contribution. Using the above method, kinetic energies for different ions were calculated and are listed in table 1. The higher kinetic energy of multiply charged ions at $563 \mathrm{~nm}$ coupled with the observation of comparatively higher charged states than $532 \mathrm{~nm}$ further proves that the phenomenon of Coulomb explosion is more facile at $563 \mathrm{~nm}$.

Previous studies carried out on Coulomb explosion phenomenon using pico/femto second laser pulses have reported anisotropic distribution of fragment ions as a function of laser polarization angle with respect to the time-of-flight axis and provided evidence regarding the role played by the transient electric field associated with the intense laser pulse. ${ }^{20-22}$ However, theoretical studies show that a multiply charged cluster can decay either by binary/ tertiary fission or by Coulomb explosion. The former is spatially anisotropic via one axis elongation of the charged cluster forming two large fragments. On the other hand, Coulomb explosion process is isotropic with small ionic fragments expanding 
Table 1. Kinetic energy of multiple charged atomic ions at 532 and $563 \mathrm{~nm}$, based on arrival time difference ' $\Delta t$ (in nanoseconds)' between forward and back-ward ion peak of mass $m$ (in amu), calculated using formula ' $E_{\text {kin }}=9.65 \times 10^{-7} \Delta t^{2} n^{2} F^{2} / 8 m$ ', where $F$ is the static electric field for ion extraction in $\mathrm{V} \mathrm{cm}^{-1}$ and $n$ is the charge of the ion.

\begin{tabular}{lccccccccc}
\hline & \multicolumn{1}{c}{ Kinetic energy (eV) } \\
\cline { 2 - 9 } Wavelength $(\mathrm{nm})$ & $\mathrm{I}^{1+}$ & $\mathrm{I}^{2+}$ & $\mathrm{I}^{3+}$ & $\mathrm{I}^{4+}$ & $\mathrm{I}^{5+}$ & $\mathrm{C}^{1+}$ & $\mathrm{C}^{2+}$ & $\mathrm{C}^{3+}$ & $\mathrm{C}^{4+}$ \\
\hline 532 & $18 \pm 2$ & $114 \pm 12$ & $376 \pm 25$ & - & - & $29 \pm 6$ & $115 \pm 30$ & $325 \pm 26$ & - \\
563 & $235 \pm 23$ & $450 \pm 32$ & $650 \pm 45$ & $*$ & $*$ & $175 \pm 30$ & $260 \pm 40$ & $*$ & $*$ \\
\hline
\end{tabular}

Kinetic energy measured at $\sim 5 \times 10^{9} \mathrm{~W} / \mathrm{cm}^{2}$. The error bars have been derived from a set of five different measurements. For some ions (marked with $*$ ) the kinetic energies could not be measured from the peak splitting method since the forward and backward component could not be separated properly

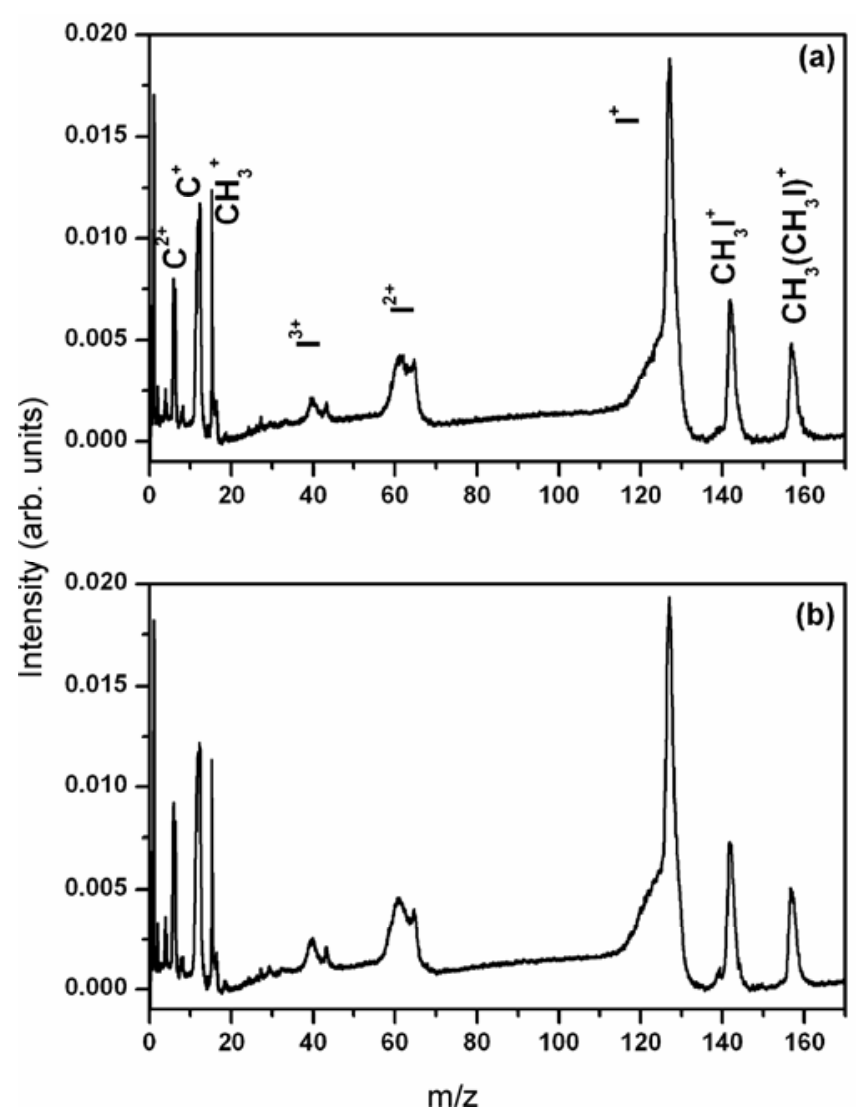

Figure 2. Time of flight mass spectra of $\mathrm{CH}_{3} \mathrm{I}$ clusters at (a) parallel $\left(0^{\circ}\right)$ and (b) perpendicular $\left(90^{\circ}\right)$ polarization at $532 \mathrm{~nm}$.

radially. ${ }^{23}$ However, these theoretical studies have been performed for high laser intensity cases $\left(10^{14}\right.$ $10^{20} \mathrm{~W} / \mathrm{cm}^{2}$ ) and no information is available regarding the spatial distribution of fragment ions in case of nanosecond Coulomb explosion. Therefore, in order to understand the effect of laser polarization on the phenomenon of Coulomb explosion of clusters under our experimental conditions, laser polarization dependent mass spectroscopic experiments were also carried out. Figure 2(a) and (b) illustrate time-of-flight mass spectra of $\mathrm{CH}_{3} \mathrm{I}$ clusters recorded at parallel $\left(0^{\circ}\right)$ and (b) perpendicular $\left(90^{\circ}\right)$ laser polarization with respect to the time of flight axis at $532 \mathrm{~nm}$. From the figure it can be realized that, the fragmentation pattern as well as the ion intensity distribution was found to be similar, suggesting isotropic disintegration of multiply charged $\mathrm{CH}_{3} \mathrm{I}$ cluster. It must be noted here that multiply charged ions $\mathrm{C}^{2+}, \mathrm{C}^{3+}, \mathrm{C}^{+}, \mathrm{I}^{3+}$ and $\mathrm{I}^{2+}$ (figure 2) are showing asymmetric peak profile. In case of $\mathrm{I}^{+}$ions, this asymmetry is not well-resolved due to lower kinetic energy associated with it.

Figure 3 shows kinetic energy of multiply charged ions $\mathrm{C}^{2+}, \mathrm{C}^{3+}, \mathrm{I}^{2+}$ and $\mathrm{I}^{3+}$ as a function of polarization angle. Similarly, figure 4 shows variation in ion yield of multiply charged ions as a function of laser polarization angle with respect to the time of flight axis. From these figures, it is clear that the ion yields as well as their kinetic energies are found to be independent of laser polarization angle, thus indicating that the Coulomb explosion process induced by nanosecond laser in clusters is isotropic.

To further cross check the electron heating mechanism, additional experiments were carried out using doped methyl iodide clusters. Here one must keep in mind that the dopant molecule/clusters should neither ionize nor undergo Coulomb explosion under our (giga watt intensity) experimental conditions. In these studies, water was chosen as the dopant due to its higher ionization potential $(12.62 \mathrm{eV})$ as compared to that of methyl iodide $(9.54 \mathrm{eV})$. This higher ionization potential of water decreases the multiphoton ionization probability of the water molecules/clusters as compared to that of methyl iodide molecules/clusters under identical laser excitation conditions. Further, the water has much lower vapour pressure as compared to that of methyl iodide. Hence on co-expansion, the methyl iodide clusters have minor contribution from dopant 

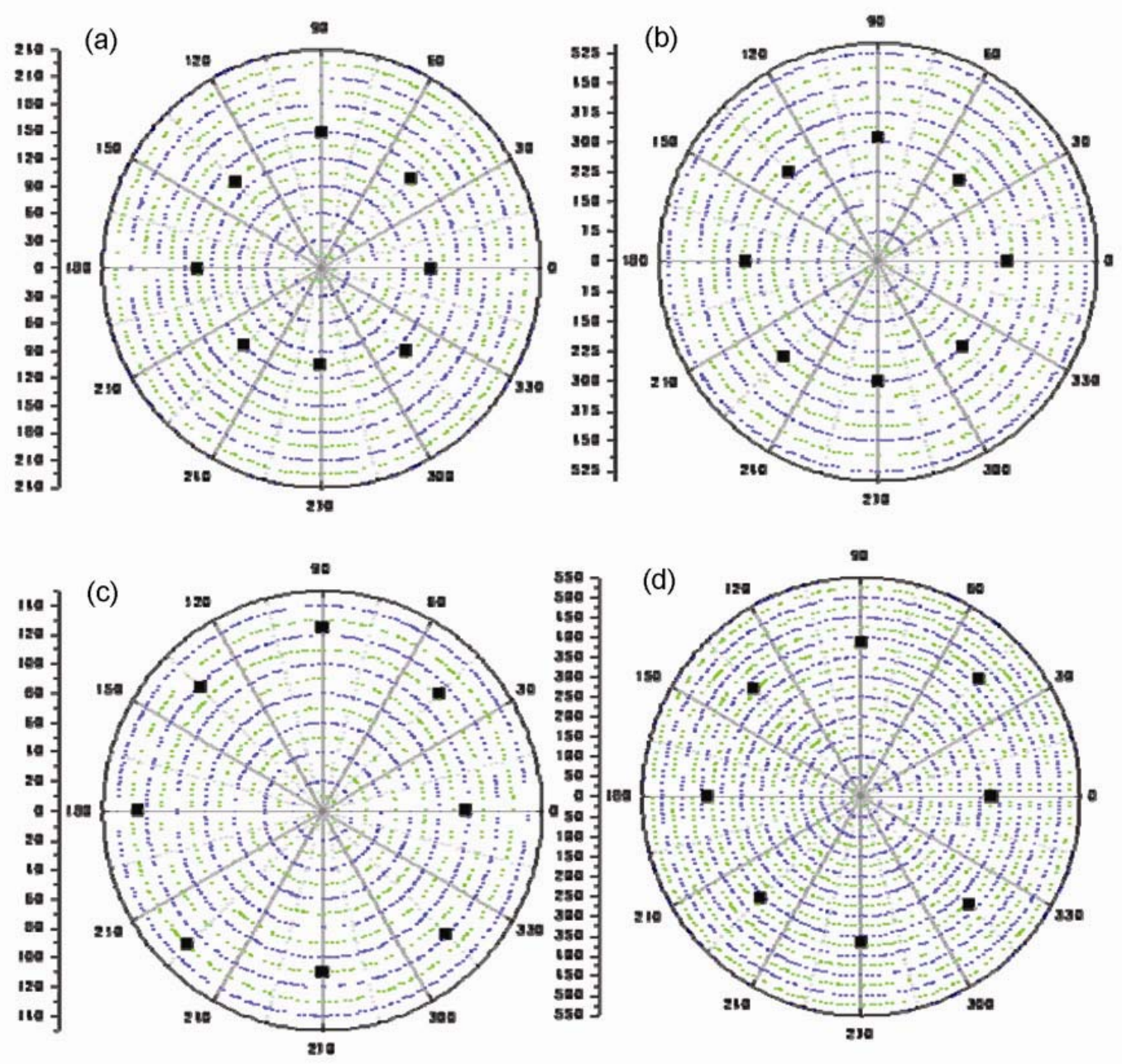

Figure 3. Variation in kinetic energy of multiply charged ions (a) $\mathrm{C}^{2+}$, (b) $\mathrm{C}^{3+}$, (c) $\mathrm{I}^{2+}$ and (d) $\mathrm{I}^{3+}$ as a function of $\lambda / 2$ plate rotation.

water molecules. As another requirement from experimental point of view, the multiply charged ions of dopant should be different from carbon and iodine and should be unambiguously assignable. Water meets all these criteria as well as it is easily available in the pure form. So water was chosen as dopant.

No ion signal arising due to interaction of water clusters with laser pulse could be observed under our experimental conditions when pure $\mathrm{H}_{2} \mathrm{O}$ clusters generated by supersonic expansion of $\mathrm{H}_{2} \mathrm{O}$ vapors at room temperature seeded in 1-5 atm pressure of the helium were subjected to $563 \mathrm{~nm}$ laser pulses of gigawatt intensity. As mentioned above, the probable explanation for this observation could be lower multiphoton ionization cross-section of water clusters at $563 \mathrm{~nm}$. However, when water doped methyl iodide clusters (generated by supersonic expansion of $\mathrm{CH}_{3} \mathrm{I}$ and water sample mixed in 9:1 ratio (by volume)) were subjected to $563 \mathrm{~nm}$ laser radiation, these doped clusters were found to undergo Coulomb explosion resulting in generation of energetic multiply charged atomic ions of oxygen (up to $\mathrm{O}^{4+}$ ) in the time-of-flight mass spectra along with other multiply charged atomic ions of carbon and iodine. Therefore, this study demonstrates that doping of methyl iodide clusters with water leads to induction of Coulomb explosion in dopant water also which now forms an integral part of methyl iodide cluster. These results show that Coulomb explosion is a collective property of the cluster as a whole and individual molecular properties do not play significant role.

\section{Discussion}

The highest charged state experimentally observed in case of $532 \mathrm{~nm}$ are limited up to $\mathrm{C}^{3+}$ and $\mathrm{I}^{3+}$ 

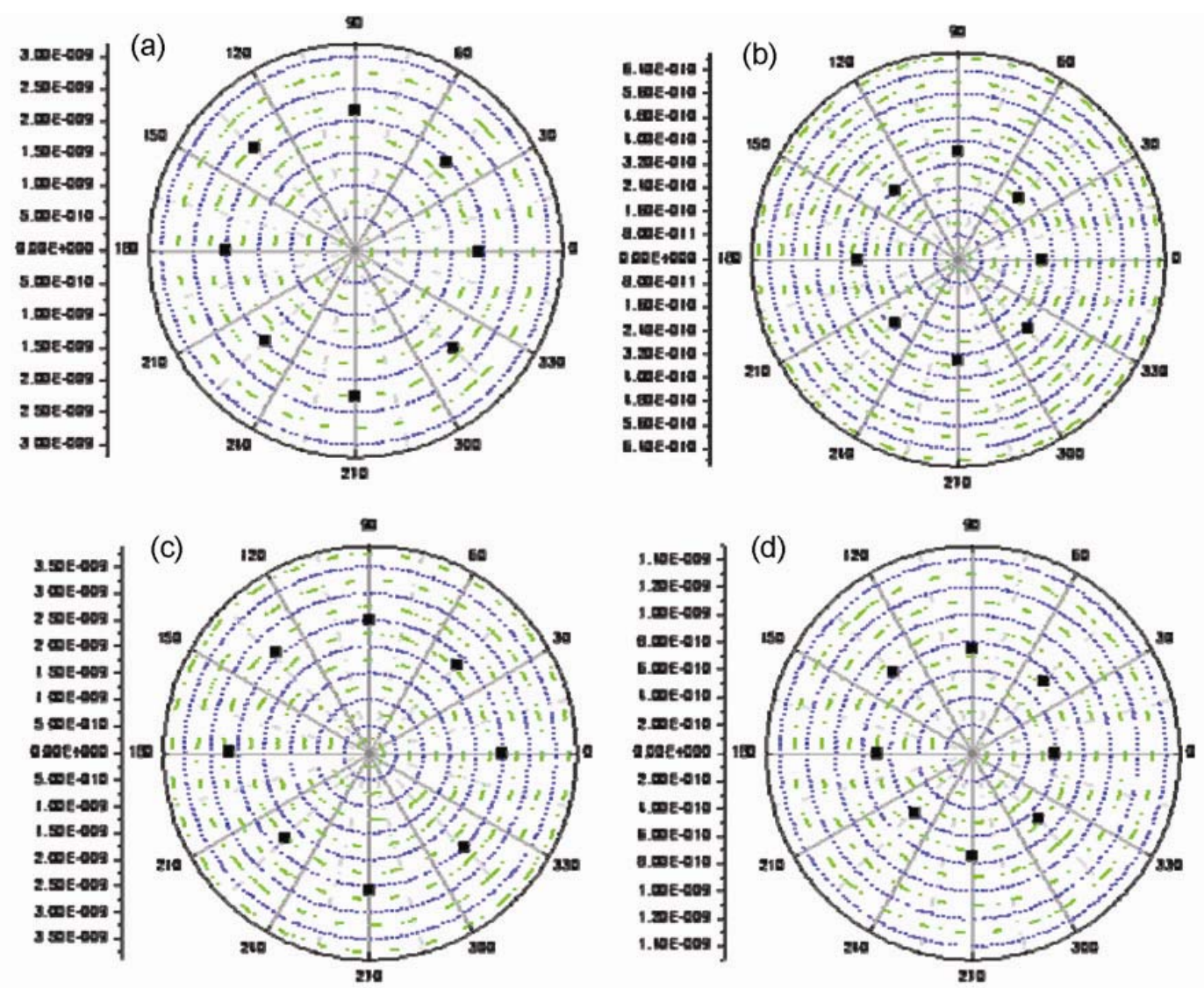

Figure 4. Variation in ion yield of multiply charged ions (a) $\mathrm{C}^{2+},(\mathbf{b}) \mathrm{C}^{3+},(\mathbf{c}) \mathrm{I}^{2+}$ and (d) $\mathrm{I}^{3+}$ as a function of $\lambda / 2$ plate rotation.

whereas in case of $563 \mathrm{~nm}$ the highest charged states observed span up to $\mathrm{C}^{4+}$ and $\mathrm{I}^{5+}$ at similar laser intensity. Thus, the experimental results clearly indicate that the charge states and the kinetic energies of atomic ions generated as a result of Coulomb explosion of methyl iodide clusters on interaction with 532 and $563 \mathrm{~nm}$ are significantly different. Though one would expect that the order of charged states and extent of kinetic energy release should be somewhat higher for $532 \mathrm{~nm}$, which has higher photon energy compared to $563 \mathrm{~nm}$, but the experimental observation are contrary to this common perception. The multiphoton ionization probability of methyl iodide clusters is not expected to be very different at 532 and $563 \mathrm{~nm}$ since at both these wavelengths five photons are required for ionization (assuming that ionization potential of methyl iodide clusters is $\leq 9.5 \mathrm{eV}$ ). Hence, our experimental observation cannot be explained based on mere multiphoton absorption of photons by the cluster. If one assumes multiphoton ionization to be the sole process re- sponsible for generation of multiply charged ions, the intensity threshold for the appearance of these multiply charged ions can be calculated by using the formula of Auguste et $a l^{16}-{ }^{\prime} I\left(\mathrm{~W} / \mathrm{cm}^{2}\right)=$ $4 \times 10^{9} E_{i}^{4} / Z^{2}$, where $E_{i}$ is ionization potential of the multiply charged ion $(\mathrm{eV})$ and $Z$ is the charge on the ion. Using the above equation, the intensity threshold for appearance of multiply charged ions is found to be between $10^{14}$ and $10^{15} \mathrm{~W} / \mathrm{cm}^{2}$, which is at least five orders of magnitude higher than the intensity used in our experiments. Though, the concept of ponderomotive heating of electrons liberated upon ionization of clusters could, to some extent, explain the results, as the ponderomotive heating of the cluster by the free electron is proportional to $I \lambda^{2}$, which suggests that the extent of energy deposited within the cluster would be higher at $563 \mathrm{~nm}$, as compared to $532 \mathrm{~nm}$.

Observation of isotropic ion yield and kinetic energy of multiply charged ions under our experimental conditions points towards IBS heating of 
cluster. In case of high intensity (pico/femto second laser-induced) Coulomb explosion of clusters, observation of contribution from two different processes to the ion energy distribution of multiply charged ions generated due to disassembly of atomic and molecular cluster has been reported. One of the processes is related to kinetic energy acquired by ions due to Coulomb repulsion and is consistent with the Coulomb's law. The other process which contributes to the kinetic energy of these ions is the electric field associated with the intense laser pulse. This higher energy component is found to exhibit anisotropy with respect to the laser polarization vector. Under our experimental studies, based on Keldysh parameter ' $\gamma$ ' $\left(\gamma=\left(I_{p} / 2 U_{p}\right)^{1 / 2}\right.$, where $I_{p}$ is ionization potential and $U_{p}$ is ponderomotive energy given in $\mathrm{eV}$ by ' $U_{p}=e^{2} I \lambda^{2} /\left(8 \pi^{2} \varepsilon_{0} m_{e} c^{3}\right)=9.33 \times 10^{-14}$ $I \lambda^{2}\left(\mu \mathrm{m}^{2}\right)$, where $I$ is the intensity of laser pulse in $\left.\mathrm{W} / \mathrm{cm}^{2}\right)$ it can be assumed that the ionization within the cluster is initiated by multiphoton ionization as $\gamma \sim 180$ (since for $\gamma>>1$, ionization is attributed to MPI processes, while for $\gamma<1$ it is attributed to field ionization mechanism). Thus, the isotropic behaviour of the Coulomb explosion process suggests that the electric field associated with the giga watt

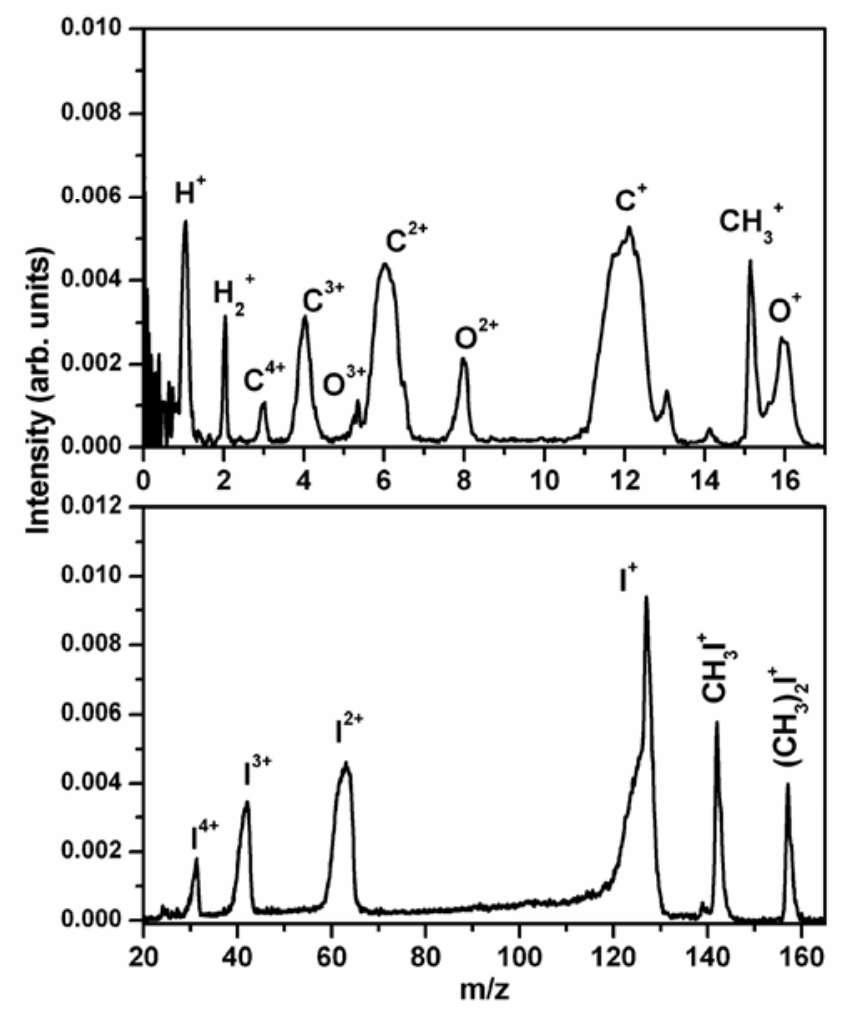

Figure 5. Time of flight mass spectra of $\mathrm{CH}_{3} \mathrm{I}-\mathrm{H}_{2} \mathrm{O}$ mixed clusters when subjected to $563 \mathrm{~nm}$ laser pulses at an intensity of $\sim 10^{9} \mathrm{~W} / \mathrm{cm}^{2}$. laser pulses does not play a dominant role in initiating the ionization process in the clusters. Thus, the ionization process which produces multiply charged atomic ions is independent of laser polarization under our experimental conditions.

Further, induction of Coulomb explosion in water molecules which were doped in methyl iodide clusters also suggests that the mechanism which finally results in Coulomb explosion is a collective property of the cluster as a whole and does not discriminate between the constituents of the cluster. As stated above, this could be the electron heating of the cluster, as a consequence of absorption of laser energy by electrons upon collision with neutral and ionic species within the cluster. The concept of electron heating of molecular cluster under the influence of nanosecond laser pulse is further supported by measurement of kinetic energy of electrons liberated during the course of Coulomb explosion. Using retarding field analyzer, in our earlier study at $5 \times 10^{9} \mathrm{~W} / \mathrm{cm}^{2}$ intensity, ${ }^{12}$ we reported detection of electrons with most probable and maximum kinetic energy of $\sim 5$ and $20 \mathrm{eV}$ liberated during the course of Coulomb explosion of methyl iodide clusters at $532 \mathrm{~nm}$. Recently, Zhang et $a l^{18}$ have also reported detection of electrons with mean and maximum kinetic energy of $\sim 17$ and $30 \mathrm{eV}$ upon Coulomb explosion, when benzene clusters were irradiated with $532 \mathrm{~nm}$ laser pulse having intensity $\sim 9.3 \times$ $10^{9} \mathrm{~W} / \mathrm{cm}^{2}$.

Before concluding we would like to discuss about the crucial role played by the excited electronic states of the molecular system in inducing Coulomb explosion in clusters, which in our opinion plays a vital role in induction of Coulomb explosion at low laser intensities. This is because; the primary initiation step for Coulomb explosion i.e. initial multiphoton ionization of the cluster itself is dependent on the nature and energy level of electronic excited states of the molecular system. Our previous studies carried out on several other molecular systems have suggested that multiphoton excitation to intermediate excited electronic states play a vital role in inducing Coulomb explosion in clusters. ${ }^{12,14,15}$ The multiphoton excitation/ionization probability, leading to generation of electrons for subsequent IBS heating of the cluster would depend on the order of multiphoton process. If some resonant intermediate state is involved, the ionization probability of the molecular cluster system would be enhanced significantly which will reflect itself in lower threshold intensity for observation of multiply charged ions. This asser- 
tion is also supported by the experimental results of H. Li and co-workers, ${ }^{18}$ whereby they have carried out Coulomb explosion studies on methyl iodide and benzene clusters at 532 and $1064 \mathrm{~nm}$. In both these studies, though the order of multiply charged ions obtained was higher at $1064 \mathrm{~nm}$ as compared to $532 \mathrm{~nm}$, but the threshold intensity for initiation of Coulomb explosion in these clusters was two order of magnitude higher for $1064 \mathrm{~nm}$ wavelength as compared to that of $532 \mathrm{~nm}$. Thus, one can say that resonant intermediate state can act as doorway steps for facile Coulomb explosion in giga-watt intensity conditions.

\section{Conclusion}

In conclusion, we have carried out nanosecond laserinduced Coulomb explosion studies at 532 and $563 \mathrm{~nm}$ which are expected to have comparable multiphoton ionization probabilities for methyl iodide clusters under similar laser intensity conditions. Observation of higher charged states at $563 \mathrm{~nm}$ compared to $532 \mathrm{~nm}$ for Coulomb exploded atomic ions, supports the preposition of IBS heating of the ionized molecular system under the influence of the laser pulse. Further, isotropic behaviour of the multiply ionized atomic ions as a function of laser polarization, coupled with induction of Coulomb explosion of the dopant water molecules having higher ionization potential as compared to that of its host matrix i.e. methyl iodide, suggests that the mechanism leading to Coulomb explosion is a collective property of the cluster as a whole and does not discriminate between the constituents of the cluster. The present studies clearly show that though the recently proposed three-stage model comprising of 'multiphoton ionization, ignited-inverse bremsstrahlung heating and electron impact ionization' could qualitatively explain the experimental observations of Coulomb explosion at giga watt laser intensities, but a much more detailed experimental and theoretical study is clearly needed before a comprehensive picture of the nanosecond laserinduced Coulomb explosion could emerge. In addition, the crucial role of multiphoton excited resonant electronic states which directly influences the ionization probability of the clusters, as well as the role of clusters size essential for confinement of the ionized electron for the duration of the laser pulse to have efficient extraction of energy from the laser field cannot be overlooked.

\section{Acknowledgements}

The authors are thankful to Dr A Majumder, Laser and Plasma Technology Division, BARC for many fruitful discussions and comments on the manuscript. Dr T Mukherjee, Director, Chemistry Group and $\operatorname{Dr} \mathrm{D}$ Das, Head, Chemistry Division are acknowledged for their keen interest and continuous support for this work.

\section{References}

1. Castleman Jr A W and Keesee R G 1988 Science 241 36

2. Murphy B F, Hoffmann K, Belolipetski A, Keto J and Ditmire T 2008 Phys. Rev. Lett. 101203401

3. Kumarappan V, Krishnamurthy M and Mathur D 2003 Phys. Rev. A67 0632071

4. Krainov V P and Smirnov M B 2002 Phys. Rep. 370 237

5. Ford J V, Zhong Q, Poth L and Castleman Jr A W 1999 J. Chem. Phys. 1106257

6. Ford J V, Poth L, Zhong Q and Castleman Jr A W 1999 Int. J. Mass Spectrom. 192327

7. Wabnitz $\mathrm{H}$ et al 2002 Nature 420482

8. Ditmire T, Tisch J W G, Springate E, Mason M B, Hay N, Smith R A, Marangos J and Hutchinson M H R 1997 Nature 38654

9. Donnelly T D, Ditmire T, Neuman K, Perry M D and Falcon R W 1996 Phys. Rev. Lett. 762472

10. Mangles S P D 2004 Nature 431535

11. Ditmire T, Zweiback J, Yanovsky V P, Cowan T E, Hays G and Wharton K B 1999 Nature 398489

12. Sharma P, Vatsa R K, Kulshreshtha S K, Jha J, Mathur D and Krishnamurthy M $2006 \mathrm{~J}$. Chem. Phys. 125 034304

13. Luo X, Niu C, Kong X, Wen L, Liang F, Pei K, Wang B and Li H 2005 Chem. Phys. 31017

14. Sharma P and Vatsa R K 2007 Rapid Commun. Mass Spectrom. 212663

15. Sharma P and Vatsa R K 2008 Euro. Phys. Lett. 84 43003

16. Auguste T, Monot P, Mainfray G and Manus C $1992 \mathrm{~J}$. Phys. B: At. Mol. Opt. Phys. 254181

17. Wang W, Li H, Niu D, Wen L and Zhang N 2008 Chem. Phys. 352111

18. Zhang N, Wang W, Cang H, Wang H and Li H 2009 Chem. Phys. Lett. 46914

19. Sharma P and Vatsa R K 2009 Curr. Appl. Phys. 9140

20. Symes D R, Hohenberger M, Henig A and Ditmire T 2007 Phys. Rev. Lett. 98123401

21. Kumarappan V, Krishnamurthy M and Mathur D 2001 Phys. Rev. Lett. 87085005

22. Sakbe S et al 2001 Phys. Plasmas $\mathbf{8} 2517$

23. Last I, Levy Y and Jortner J 2005 J. Chem. Phys. 123 154301 